\title{
Stress Power Dependent Self-Heating Degradation of Metal-Induced Laterally Crystallized n-Type Polycrystalline Silicon Thin-Film Transistors
}

\author{
Huaisheng Wang, Mingxiang Wang, Member, IEEE, Zhenyu Yang, Han Hao, and Man Wong, Senior Member, IEEE
}

\begin{abstract}
Self-heating degradation of n-type metal-induced laterally crystallized polycrystalline silicon thin-film transistors is systematically investigated under various stress powers. A two-stage degradation behavior with turnaround effect at the initial stage is characterized. The initial degradation stage is related to breaking of weak $\mathrm{Si}-\mathrm{H}$ bonds. The floating-body effect by released hydrogen ions is responsible for the observed backshift of the transfer curve during the initial stress. On the other hand, the normal degradation stage occurs by breaking of strong $\mathrm{Si}-\mathrm{Si}$ bonds and trap generation at grain boundaries (GBs) and the gate oxide/channel interface. Our model is supported by observed different activation energies related to two degradation stages and a direct observation of the continuous increase in GB trap density during the normal degradation. Furthermore, during the normal degradation stage, an anomalous continuous field-effect mobility increase along with its $V_{g}$ dependence shift is first observed. It is clarified that this behavior is not a true channel mobility increase, but a consequence of stress-related trap generation.
\end{abstract}

Index Terms-Field-effect mobility, metal-induced lateral crystallization, polycrystalline silicon (poly-Si), self-heating (SH) degradation, thin-film transistors (TFTs).

\section{INTRODUCTION}

$\mathbf{L}$ OW-TEMPERATURE polysilicon (LTPS) thin-film transistors (TFTs) have attracted much attention for their potential for system-on-panel applications such as full integrated active matrix displays by liquid crystal or by organic light emitting diodes [1]. Although excimer laser crystallization (ELC) is currently a widely employed LTPS technology [2]-[5], recently, metal-induced lateral crystallization (MILC) has been demonstrated as a promising LTPS technology with some unique advantages over other techniques [6]. Currently, highperformance polycrystalline silicon (poly-Si) TFTs have been fabricated by using various technologies [6], [7]. However, device reliability still remains a limiting factor for poly-Si TFTs toward future application in large-scale integration circuits. It is

Manuscript received August 13, 2007; revised September 14, 2007. This work was supported by the National Natural Science Foundation of China under Contract 60406001 and in part by the Research Grants Council of the Hong Kong Special Administrative Region. The review of this paper was arranged by Editor J. Suehle.

H. Wang, M. Wang, Z. Yang, and H. Hao are with the Department of Microelectronics, Soochow University, Suzhou 215021, China (e-mail: Mingxiang_wang@suda.edu.cn).

M. Wong is with the Department of Electronic and Computer Engineering, The Hong Kong University of Science and Technology, Kowloon, Hong Kong.

Color versions of one or more of the figures in this paper are available online at http://ieeexplore.ieee.org.

Digital Object Identifier 10.1109/TED.2007.908907 particularly true for MILC TFTs since, up to now, there are still rarely reported studies on their device reliability [8], [9].

It has been identified for poly-Si TFTs that hot carrier (HC) stress [4], [5], [9]-[14] and self-heating (SH) stress [4], [7], [9], [14]-[18] are two key mechanisms inducing device degradation. In this paper, time-dependent SH degradation behaviors of n-type MILC TFTs under different stress powers were systematically investigated. Channel temperature rise in stressed devices was simulated by the finite-element method. Two features in device degradation that are different from those previously observed for ELC TFTs, namely, two-stage degradation with a turnaround effect and anomalous field-effect mobility degradation, were observed. A unified model was proposed to understand the observed degradation behaviors.

\section{EXPERIMENTS}

TFTs used in this study were in conventional self-aligned top-gate structure. First, a 50-nm amorphous Si (a-Si) was deposited on an oxidized silicon wafer by low-pressure chemical vapor deposition (LPCVD). After patterning of a-Si active islands and LPCVD of an 80-nm low-temperature oxide (LTO), a crystallization-inducing window was opened through the LTO, and 5-nm Ni was evaporated by electron beam at room temperature. The wafers were subsequently annealed for metalinduced unilateral crystallization of active islands at $550{ }^{\circ} \mathrm{C}$ for $24 \mathrm{~h}$ in $\mathrm{N}_{2}$ ambient. After $\mathrm{Ni}$ removal, another extended anneal was done at $550{ }^{\circ} \mathrm{C}$ for $24 \mathrm{~h}$. Then, LTO was removed, and another 100-nm LTO layer was deposited as gate oxide, followed by LPCVD of 300-nm poly-Si as gate. After gate patterning, a self-aligned phosphorous implantation with a dose of $4 \times 10^{15} \mathrm{~cm}^{-2}$ was introduced to form source and drain, and subsequently activated at $620^{\circ} \mathrm{C}$ for $3 \mathrm{~h}$. Contact holes were then opened before aluminum layer sputtering and patterning. Finally, wafers were sintered in forming gas.

MILC TFTs with gate width/length $\mathrm{W} / \mathrm{L}=10 / 6 \mu \mathrm{m}$ are stressed and characterized before and after stress by using Agilent 4156C semiconductor parameter analyzer and Vector MX-1100B prober. From transfer curves measured at $V_{\mathrm{ds}}=$ $0.1 \mathrm{~V}$, device characteristics such as threshold voltage $V_{\mathrm{th}}$, on current $I_{\mathrm{on}}$, field-effect mobility $\mu_{\mathrm{FE}}$, and subthreshold slope $S S$ are extracted. $I_{\text {on }}$ is defined at a gate voltage $V_{g}=12 \mathrm{~V}$. $V_{\mathrm{th}}$ is determined by linear extrapolation of $I_{d}$ to zero. The uncertainty of $V_{\mathrm{th}}$ extraction is within $0.2 \mathrm{~V}$. Devices are characterized both in the normal and reverse modes. In the reverse mode, device source and drain electrodes are reversely 


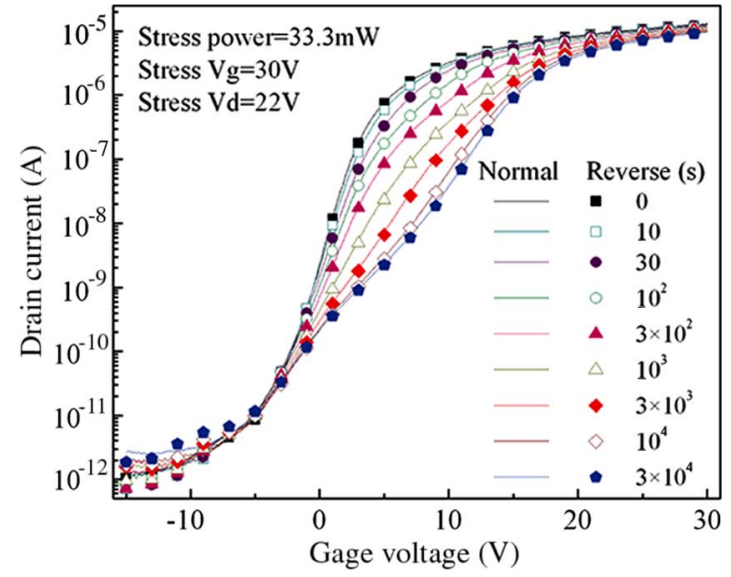

Fig. 1. Typical time evolution of transfer characteristic under SH stress condition for an n-channel MILC TFT, measured at $V_{\mathrm{ds}}=0.1 \mathrm{~V}$. Lines and symbols are for normal and reverse measurement mode, respectively.

assigned with respect to those in the normal mode. All devices for stress test have similar $V_{\mathrm{th}}$ of around $5 \mathrm{~V}$.

$\mathrm{SH}$ conditions are set with fixed stress $V_{g}$ of 30 or $35 \mathrm{~V}$, varied stress $V_{d}$ from 16 to $22 \mathrm{~V}$, and resulting stress power from $\sim 21$ to $\sim 33 \mathrm{~mW}$. Application of such bias stresses avoids $\mathrm{HC}$ generation by impact ionization near the drain, so that only $\mathrm{SH}$-induced degradation effect is investigated. Device degradation after being stressed for varied duration from 1 to $10^{5} \mathrm{~s}$ is characterized by a percentile change in $I_{\mathrm{on}}, V_{\mathrm{th}}, S S$, and $\mu_{\mathrm{FE}}$ with respect to their initial values, i.e., $\Delta I_{\mathrm{on}}, \Delta V_{\mathrm{th}}$, $\Delta S S$, and $\Delta \mu_{\mathrm{FE}}$, respectively.

\section{Results And Discussions}

\section{A. General Degradation Characteristics}

Typical time evolutions of the transfer characteristic of an MILC TFT under SH stress, measured in both forward and reverse modes, are shown in Fig. 1. For low and high power stresses, it is seen that the most dramatic degradation occurs at the pseudosubthreshold region [19], clearly resulting in $S S$, $V_{\mathrm{th}}$, and $I_{\mathrm{on}}$ degradation. $I_{\mathrm{on}}$ degradation is reduced at the higher $V_{g}$ region due to the $V_{g}$-induced GB barrier lowering effect [20]-[22]. It is noted that $I_{\mathrm{on}}$ degradation symmetrically occurs for forward and reverse measurement modes, implying that such $\mathrm{SH}$ stress biases cause uniform degradation along the entire TFT channel. This is reasonable since no saturation occurs in our SH bias stresses. Not surprisingly, more degradation is observed under higher stress power condition. Leakage current increases with stress time, under low and high power stresses, and observed in forward and reverse modes. It implies stress-induced trap state generation near the source and drain junctions.

Percentile degradations of $I_{\mathrm{on}}$ and $V_{\mathrm{th}}$ against stress time under different stress powers from 21.1 to $33 \mathrm{~mW}$ are shown in Fig. 2(a) and (b), respectively. From the dependences of $\Delta V_{\mathrm{th}}$ and $\Delta I_{\text {on }}$ and for all stress power, it is seen that there is an initial degradation stage in which device characteristics remain little changed. This initial stage of stress remarkably varies from $\sim 1$ to $\sim 10^{3}$ s with decreasing stress power. Significant decrease

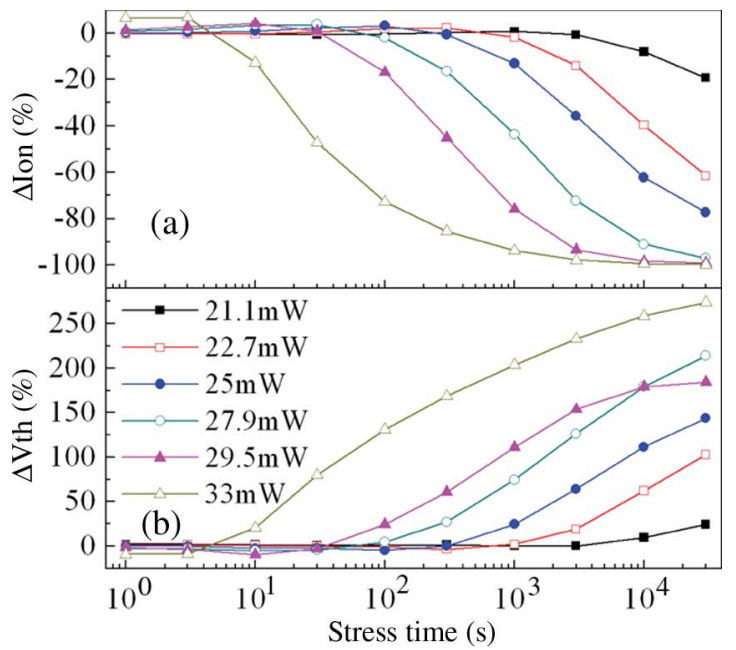

Fig. 2. Dependence of (a) on current and (b) threshold voltage degradation on stress time under various stress powers.
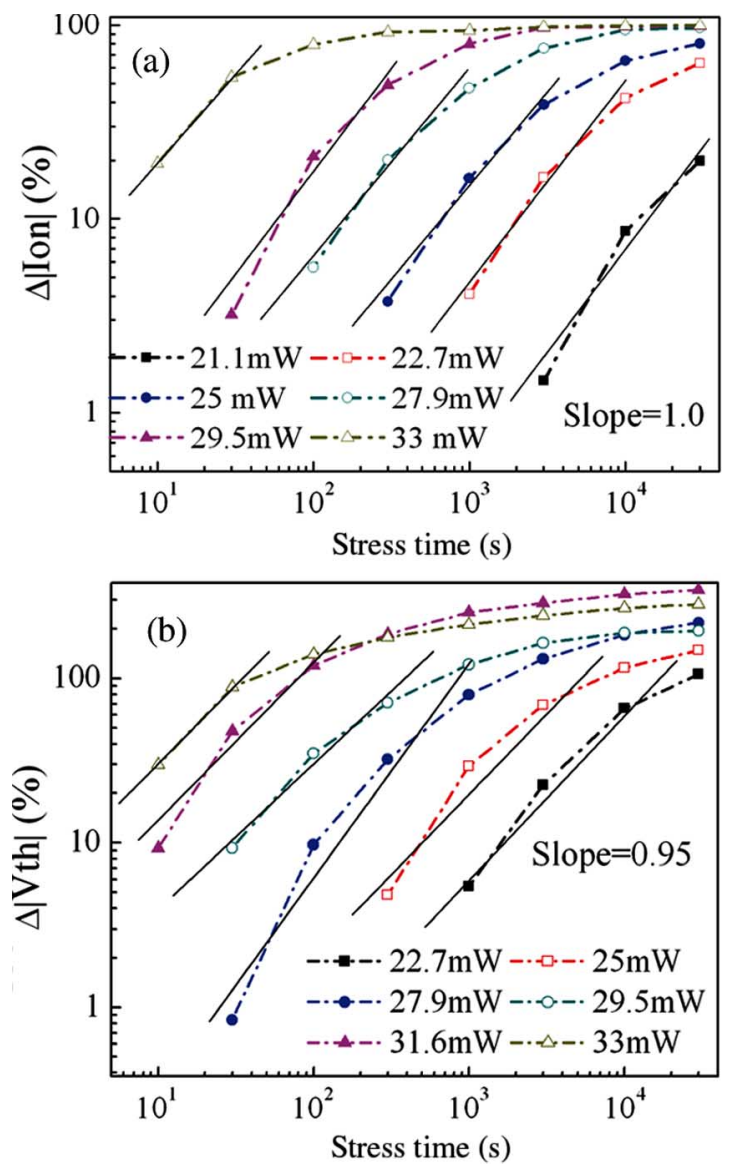

Fig. 3. Dependence of (a) $\left|\Delta I_{\text {on }}\right|$ and (b) $\left|\Delta V_{\text {th }}\right|$ after initial stage on stress time plotted in logarithmic scale for various stress power conditions. The degradation slopes are 1.0 and 0.95 , respectively.

in $I_{\mathrm{on}}$ and increase in $V_{\mathrm{th}}$ continuously occur only after the initial stage. We then define it as the normal degradation stage.

$I_{\mathrm{on}}$ and $V_{\text {th }}$ degradation characteristics after the initial stage plotted in logarithmic scale are shown in Fig. 3(a) and (b), respectively. Average degradation slopes are determined to be 1.0 and 0.95 for data range $\left|\Delta I_{\mathrm{on}}\right| \lesssim 50 \%$ and $\left|\Delta V_{\mathrm{th}}\right| \lesssim 100 \%$, 


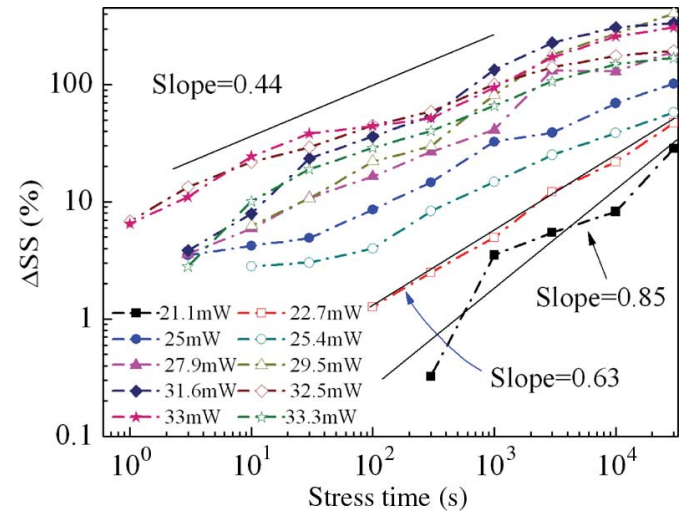

Fig. 4. Subthreshold slope degradation against stress time under various stress power conditions.

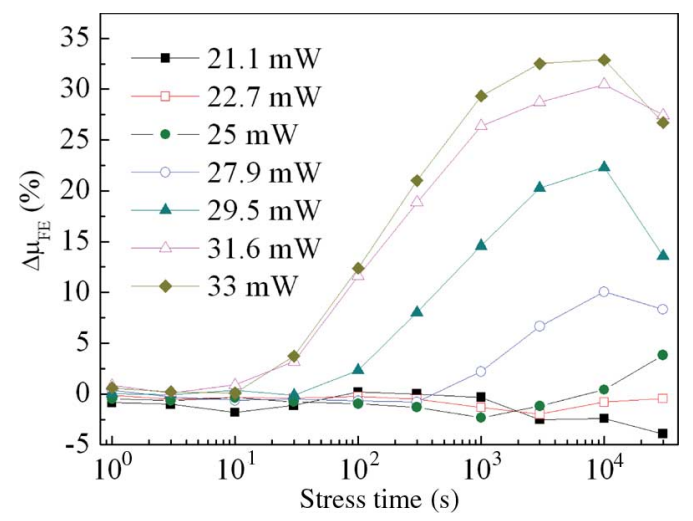

Fig. 5. Stress-time-dependent $\mu_{\mathrm{FE}}$ percentile variation under different stress power conditions.

respectively, which strongly suggests an underlying degradation mechanism of defect generation either by dissolution of $\mathrm{Si}-\mathrm{H}$ bonds [15] or breaking/distortion of $\mathrm{Si}-\mathrm{Si}$ bonds [10] at grain boundaries (GBs) and/or the gate oxide/channel interface. After long time stress, the degradation slope of $I_{\mathrm{on}}$ and $V_{\mathrm{th}}$ is seen to be lowered.

$S S$ degradation for various stress powers is shown in Fig. 4 . A power-law dependence is also observed. Degradation slope $\alpha$ for medium and high stress power conditions varies from 0.36 to 0.55 , with an average value of 0.44 , whereas for the two lowest stress powers, $\alpha$ is found to be $0.85(21.1 \mathrm{~mW})$ and 0.63 $(22.7 \mathrm{~mW})$. Hu et al. [11] demonstrated that the degradation slope for defect generation was expected to be between 0.5 (diffusion limited) and 1 (reaction limited). Our observation might indicate a transition of the mechanism from reaction limited to diffusion limited when increasing the stress power, thus enhancing the channel temperature rise. Degradation of $S S$ is a consequence of defect generation along the entire channel upon high current stress under SH [17], depending mainly on deep states at the gate oxide/channel interface and/or GBs [5], [14].

\section{B. Anomalous Field-Effect Mobility Degradation}

Different degradation behaviors of $\mu_{\mathrm{FE}}$ are found under low or high stress power conditions, as shown in Fig. 5. Under low stress power conditions, $\mu_{\mathrm{FE}}$ remains almost unchanged

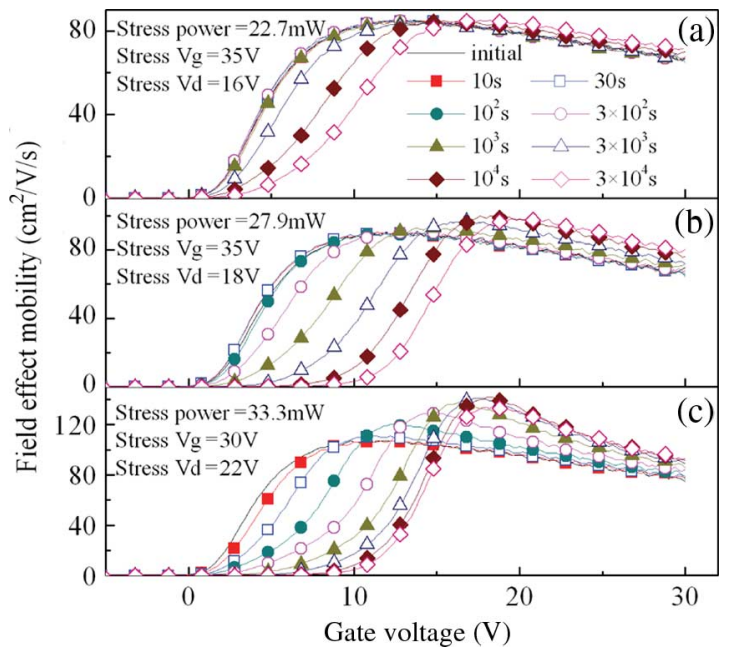

Fig. 6. Evolution of $\mu_{\mathrm{FE}}$ versus $V_{g}$ dependence upon SH stress for (a) low, (b) medium, and (c) high stress power conditions.

or just slightly decreases after long time stress. Under high stress power conditions, however, $\mu_{\mathrm{FE}}$ continuously increases with stress time until finally saturating/decreasing after $10^{4} \mathrm{~s}$ stress. The $\mu_{\mathrm{FE}}$ increase becomes more evident with increasing stress power. Besides $\mu_{\mathrm{FE}}$ or maximum transconductance $\left(g_{m_{-} \max }\right)$ variation, a continuous nearly parallel shift of $\mu_{\mathrm{FE}}$ versus $V_{q}$ dependence in the positive direction is observed. The evolution of the $\mu_{\mathrm{FE}}$ dependence upon SH stress for low, medium, and high stress power conditions is shown in Fig. 6(a)-(c), respectively. The shift is more significant for high power stress conditions, but begins to saturate after $10^{4}$-s stress synchronizing with the $\Delta \mu_{\mathrm{FE}}$ saturation (see Fig. 5). In poly-Si TFTs, $\mu_{\mathrm{FE}}$ or $g_{m_{-} \max }$ degradation was commonly observed in HC degradation [5], [10]-[12]. Previously, there were observations of an initial increase in $\mu_{\mathrm{FE}}$ or $g_{m_{-} \max }$ under $\mathrm{HC}$ stress due to the channel shortening effect by $\overline{\mathrm{H} C}$ injection into the gate oxide at the drain end [10], [12], [13]. However, to our knowledge, continuous $\mu_{\mathrm{FE}}$ or $g_{m_{-} \text {max }}$ increase in poly-Si TFTs upon SH stress was not previously reported.

The parallel shift of the $\mu_{\mathrm{FE}}$ dependence is found to be directly related to the stress-induced device $V_{\mathrm{th}}$ degradation. This is clearly demonstrated in Fig. 7, in which the time evolution of $\mu_{\mathrm{FE}}$ dependence at different stress powers is plotted against $V_{g}-V_{\text {th }}$ instead of $V_{g}$ itself, where $V_{\text {th }}$ degrades with stress time. Hence, stress-induced $V_{\text {th }}$ shift is deducted from $V_{g}$ dependence of $\mu_{\mathrm{FE}}$. Strikingly, all such plotted $\mu_{\mathrm{FE}}$ curves at different stress times almost coincide with each other at their rising and falling stages, and there is no " $V_{g}$ dependence" shift anymore. In poly-Si TFTs, the device $V_{\text {th }}$ is determined to be the $V_{g}$ under which sufficient increase in channel mobility rather than channel charge inversion is obtained. [9], [19]. Therefore, this correlation of the $\mu_{\mathrm{FE}}$ shift with $V_{\mathrm{th}}$ degradation can be explained by the stress-induced GB trap generation, which delays the increase in channel carrier mobility, similar to our previous observation for MILC poly-Si TFTs under HC stress [9].

To clarify the nature of the anomalous $\mu_{\mathrm{FE}}$ increase, effective channel mobility $\mu_{\text {eff }}$ at different stress times is also extracted 


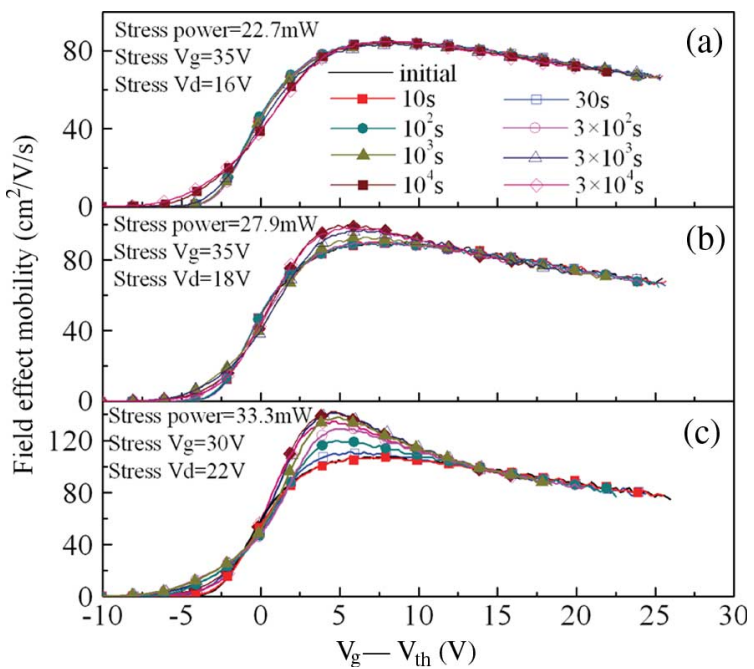

Fig. 7. Evolution of $\mu_{\mathrm{FE}}$ versus $V_{g}-V_{\mathrm{th}}$ dependence upon SH stress for (a) low, (b) medium, and (c) high stress power conditions, indicating that the $\mu_{\mathrm{FE}}$ parallel shift is directly related to device $V_{\mathrm{th}}$ degradation.

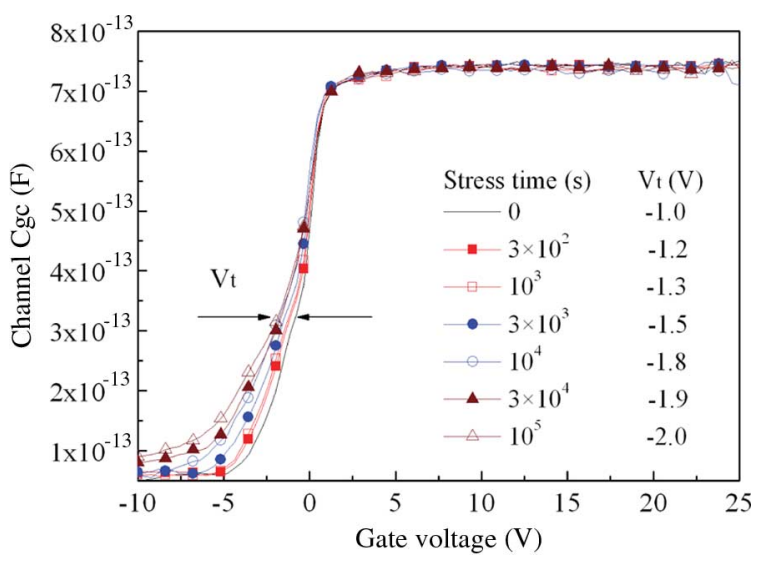

Fig. 8. Gate-to-channel capacitance versus $V_{g}(C V)$ curve for various stress times measured on a device of $\mathrm{W} / \mathrm{L}=220 / 10 \mu \mathrm{m}$. Channel inversion voltage $V_{t}$ determined by the $C V$ is listed.

through the linear region ON-state drain current by

$$
I_{d}=\frac{W}{L} \mu_{\mathrm{eff}} Q_{\mathrm{inv}} V_{d}=\frac{W}{L} \mu_{\mathrm{eff}} C_{\mathrm{ox}} V_{\mathrm{gt}} V_{d}
$$

where $V_{\mathrm{gt}} \equiv V_{g}-V_{t}$, and $V_{t}$ is the channel inversion voltage within grains. In (1), it is noted that $V_{t}$ should also degrade with stress time. A separate $C V$ measurement is carried out on a large reference device $(\mathrm{W} / \mathrm{L}=220 / 10)$ with the same fabrication process to determine its variation. The SH stress condition is set to be $V_{g}=V_{d}=25 \mathrm{~V}$, with stress power of $250 \mathrm{~mW}$. $C V$ curves at different stress times are shown in Fig. 8. $V_{t}$ is determined from the $V_{g}$ position at one third of the transition edge from depletion to inversion [19], and is listed in the figure. As demonstrated, the transition edge for channel inversion becomes more gradual with increasing stress time. As a result, $V_{t}$ is slightly degraded from $-1.0 \mathrm{~V}$ at initial to $-2.0 \mathrm{~V}$ after $10^{5}$-s stress. Since here, $V_{t}$ is the inversion voltage within grains, it is reasonable to assume the same $V_{t}$ value for other TFTs with different W/L from the reference device. Furthermore, the stress conditions for the reference TFT are also com-

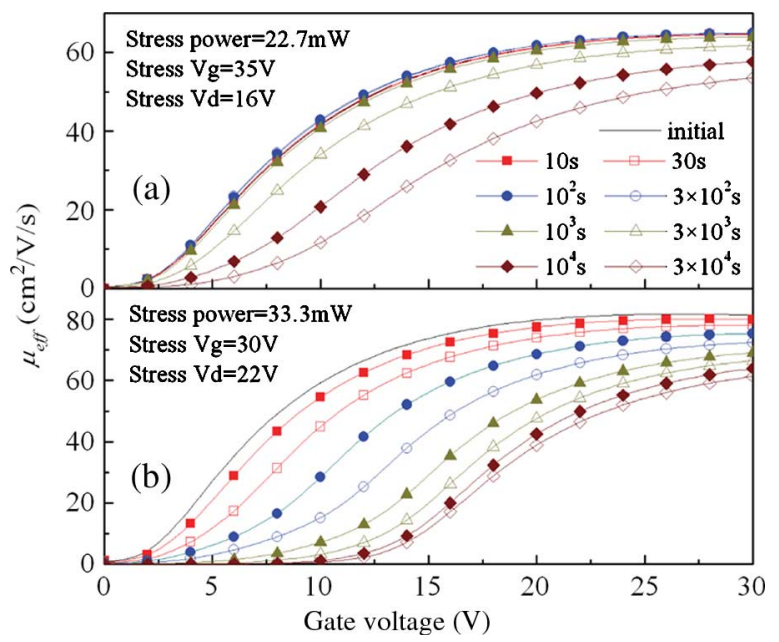

Fig. 9. Evolution of effective channel mobility $\mu_{\text {eff }}$ versus $V_{g}$ dependence under (a) low or (b) high stress power condition.

parable to those applied on the devices investigated. Therefore, it is approximated that $V_{t}$ is little degraded (within $\sim 1 \mathrm{~V}$ ) for the SH conditions investigated. Given the estimated $V_{t}$ values obtained from the $C V$ measurement on the reference device, from (1), $\mu_{\text {eff }}$ of devices stressed under low- or high-power $\mathrm{SH}$ conditions is obtained and is plotted against $V_{g}$ in Fig. 9. With no surprise, similar to that of $\mu_{\mathrm{FE}}$, the dependence of $\mu_{\mathrm{eff}}$ also continuously shifts in the positive direction with stress time. However, $\mu_{\text {eff }}$ monotonically decreases with stress time and more severely decreases under higher power stress conditions. That is, the observed $\mu_{\mathrm{FE}}$ increase is purely a transconductance increase and not a carrier mobility increase at all. Previous models attribute $g_{m_{-} \max }$ variation to the intragrain carrier mobility degradation caused by the stress-induced degradation of intragrain crystallinity [3], or to the channel shortening effect [10] by carrier trapping. However, both models fail to explain the current observation of stress-induced simultaneous $g_{m_{-}} \max$ increase and $\mu_{\text {eff }}$ decrease.

By definition, $\mu_{\mathrm{FE}}$ is conventionally calculated from $g_{m_{-} \max }$. Considering the definition of $\mu_{\mathrm{eff}}$, one can obtain the following relationship [23]:

$$
\mu_{\mathrm{FE}}=\mu_{\mathrm{eff}}+V_{\mathrm{gt}}^{*} \partial \mu_{\mathrm{eff}} / \partial V_{g}
$$

where $V_{\mathrm{gt}}^{*}$ is the $V_{\mathrm{gt}}$ value corresponding to $g_{m_{-} \max }$. In (2), it is seen that besides $\mu_{\text {eff }}$, a differential term is also included in $\mu_{\mathrm{FE}}$. Under low stress power condition, the $\mu_{\mathrm{eff}}$ decrease can be compensated by the increase in both $V_{\mathrm{gt}}^{*}$ and the differential; therefore, $\mu_{\mathrm{FE}}$ is a little degraded. Under high stress power conditions, $V_{\mathrm{gt}}^{*}$ increases much more significantly; the increase in the second term in (2) even overwhelms the decrease in $\mu_{\text {eff }}$, leading to the observed continuous $\mu_{\mathrm{FE}}$ increase with stress. Furthermore, when the shift of $\mu_{\mathrm{FE}}$ versus $V_{g}$ dependence saturates after $10^{4}$-s stress, synchronically, $\mu_{\mathrm{FE}}$ ceases to increase and finally begins to decrease [Fig. 6(c)]. As shown by (2), $\mu_{\mathrm{FE}}$ is not an independent physical variable in poly-Si TFT. In fact, $\mu_{\mathrm{FE}}$ characteristics can be mathematically rebuilt from $\mu_{\mathrm{eff}}$ data based on (2).

According to mobility models in poly-Si TFTs [20]-[24], and neglecting carrier mobility degradation by channel 


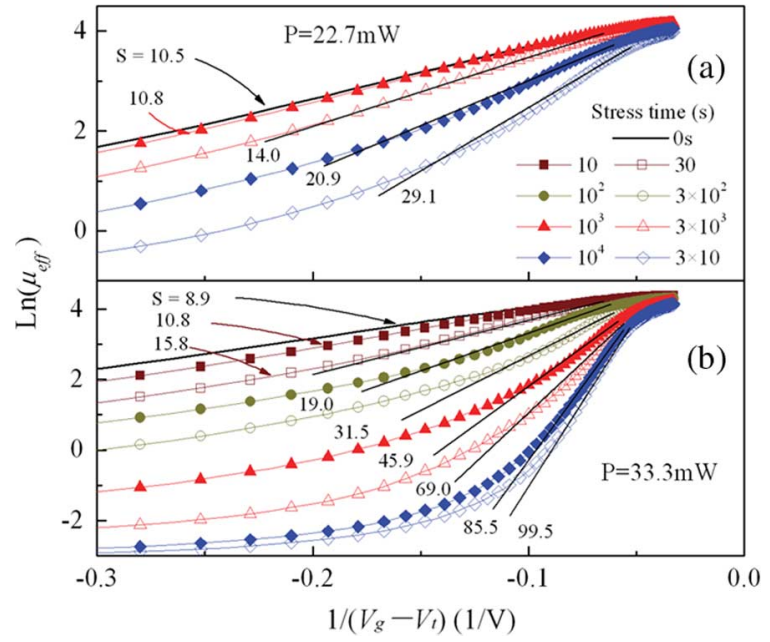

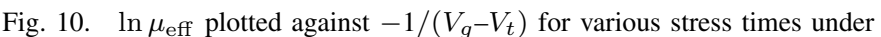
(a) low or (b) high stress power conditions. GB parameter $S$ is extracted from the slope of the plot and listed in the figure.

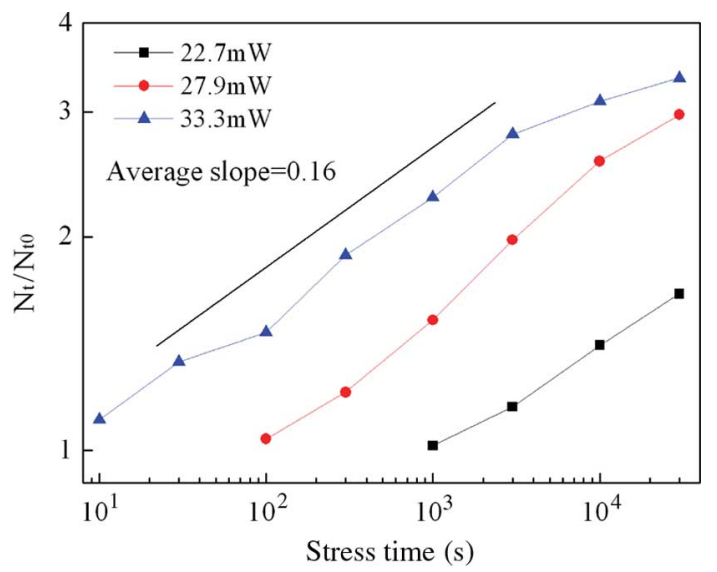

Fig. 11. GB areal trap density $N_{t}$ normalized by unstressed value $N_{t 0}$ depends on stress time in logarithmic scale for three different stress power conditions. $N_{t}$ increases with time following a power-law dependence. $N_{t 0}$ is estimated $\sim 7 \times 10^{11} \mathrm{~cm}^{-2}$ using channel layer thickness $d=50 \mathrm{~nm}$.

transverse electrical field [23], [24], $\mu_{\mathrm{eff}}$ can be expressed as

$$
\mu_{\mathrm{eff}}=\mu_{0} \exp \left(-S / V_{\mathrm{gt}}\right)
$$

where $S$ is a parameter that is related to the GB trap established potential barrier [23], [24]. Assuming monoenergetic traps of areal density $N_{t}$ on GBs, $S$ can be approximated as

$$
S=q^{3} N_{t}^{2} d / 8 \varepsilon_{s} C_{\text {ox }} k T
$$

where $\varepsilon_{s}$ is the Si permittivity, and $d$ is the channel layer [21] or the inversion layer thickness [20], [22]. Therefore, by plotting $\ln \mu_{\text {eff }} \sim-1 / V_{\mathrm{gt}}, S$ of stressed devices at different stress times can be extracted from the slope of the plot, as shown in Fig. 10. For unstressed devices, an apparent straight line is obtained in a wide $V_{g}$ range. After long time $\mathrm{SH}$ stress, although the plot gradually deviates from a straight line, the increase in the slope (or GB barrier parameter $S$ ) is evident. As a simple estimation, $N_{t}$ at different stress times normalized by initial GB areal trap density $N_{t 0}$ is calculated according to (4) for low, median, and high power stress conditions, as plotted in Fig. 11. It is

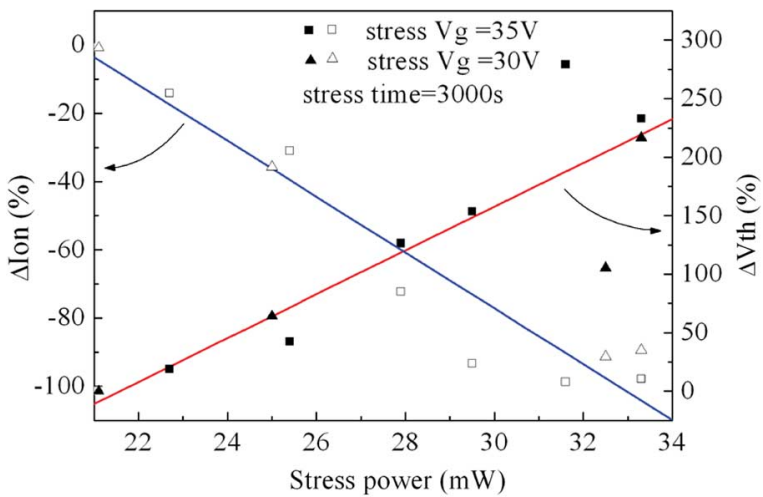

Fig. 12. Stress power dependence of $I_{\mathrm{on}}$ and $V_{\mathrm{th}}$ degradation after 3000-s stress, with stress $V_{g}$ set as 30 or $35 \mathrm{~V}$.

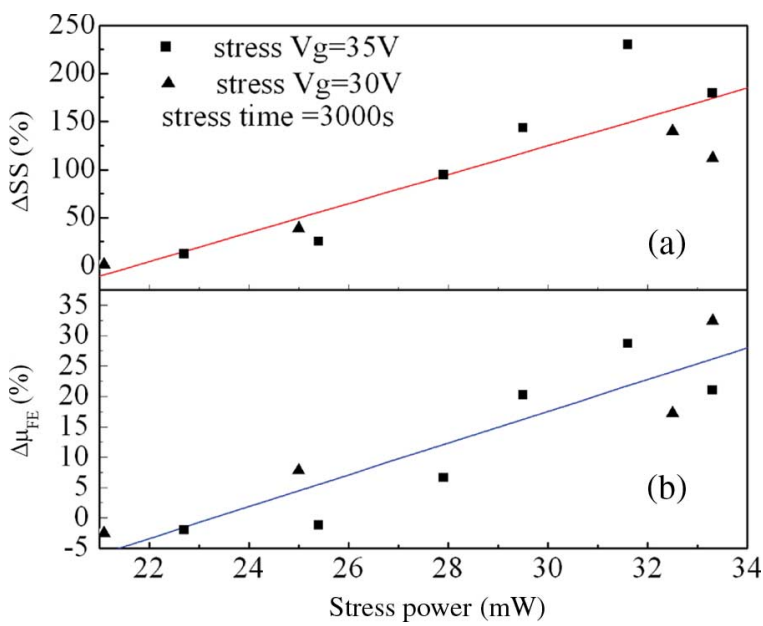

Fig. 13. Stress power dependence of (a) $S S$ and (b) $\mu_{\mathrm{FE}}$ variation after 3000 -s stress, with stress $V_{g}$ set as 30 or $35 \mathrm{~V}$.

found that $N_{t}$ increases with stress time following a power-law dependence. Obviously, more trap generation occurs for higher stress power conditions, however, with about the same powerlaw index $\alpha \approx 0.16$ for all three conditions. The increase in $N_{t}$ in $\mathrm{SH}$ conditions is a direct evidence that the defect generation mechanism at GBs is primarily responsible for the observed SH degradation. On the other hand, it is also reasonable to expect that $\mathrm{SH}$ should induce defect generation at the gateoxide/channel interface. In fact, the observation of limited distortion in the $C V$ transition edge shown in Fig. 8 should be directly related to trap generation at the gate-oxide/channel interface, to which the deviation from a straight line in Fig. 10 for long time stressed $\ln \mu_{\text {eff }} \sim-1 / V_{\text {gt }}$ plots may also be attributed. However, it is the trap generation at GBs rather than at the interface that is considered as the limiting mechanism in the currently investigated SH degradation.

\section{Two-Stage Degradation Characteristics}

The stress power dependence of device parameters degradation after 3000-s stress, such as $\Delta I_{\mathrm{on}}, \Delta V_{\mathrm{th}}, \Delta S S$, and $\Delta \mu_{\mathrm{FE}}$, is shown in Figs. 12 and 13. Clearly, all device characteristic variations correlate with stress power. Two groups of devices with fixed stress $V_{g}$ of 30 and $35 \mathrm{~V}$ are investigated. However, 
TABLE I

Thermal PARAmeters of the MATERIALS USED IN THE Simulation

\begin{tabular}{|c|c|c|c|c|c|c|c|c|c|}
\hline Material & $\begin{array}{c}\text { Density } \\
\mathrm{kg} / \mathrm{m}^{3}\end{array}$ & $\begin{array}{c}\text { Specific heat } \\
\text { capacitance } \\
\mathrm{J} /(\mathrm{kg} \cdot \mathrm{K})\end{array}$ & \multicolumn{7}{|c|}{ Thermal conductivity $\mathrm{W} /(\mathrm{m} \cdot \mathrm{K})$} \\
\hline $\mathrm{Al}$ & 2700 & 900 & \multicolumn{7}{|c|}{237} \\
\hline polysilicon & 2330 & 716.2 & \multicolumn{3}{|c|}{ 15(un-doped) } & \multicolumn{4}{|c|}{ 45( heavily doped) [25] } \\
\hline \multirow{2}{*}{ Si [26] } & \multirow{2}{*}{2330} & \multirow{2}{*}{700} & \multicolumn{2}{|c|}{$27^{\circ} \mathrm{C}$} & \multicolumn{2}{|c|}{$127^{\circ} \mathrm{C}$} & $227^{\circ} \mathrm{C}$ & \multicolumn{2}{|c|}{$327^{\circ} \mathrm{C}$} \\
\hline & & & \multicolumn{2}{|c|}{156} & 105 & & 80 & \multicolumn{2}{|r|}{64} \\
\hline \multirow{2}{*}{$\mathrm{SiO}_{2}[27]$} & \multirow{2}{*}{2200} & \multirow{2}{*}{1000} & $27.7^{\circ} \mathrm{C}$ & $59.7^{\circ} \mathrm{C}$ & $93.6^{\circ} \mathrm{C}$ & $129.2^{\circ} \mathrm{C}$ & $151.3^{\circ} \mathrm{C}$ & $177.9^{\circ} \mathrm{C}$ & $204.8^{\circ} \mathrm{C}$ \\
\hline & & & 1.30 & 1.35 & 1.39 & 1.42 & 1.47 & 1.50 & 1.53 \\
\hline
\end{tabular}

no clear difference can be discerned between the two groups. It indicates that the stress-power-decided temperature rise within the TFT channel is a dominant factor in $\mathrm{SH}$ degradation [15], [18], whereas stress $V_{g}$ seems to have no direct impact on SH degradation.

The temperature rise in stressed devices is simulated by the finite-element method using the commercial tool ANSYS. The calculation domain is a whole 4-in. wafer with a single TFT structure situated in the center; however, only the central TFT area needs to be fine meshed. The wafer bottom is fixed at a wafer chuck temperature of $27^{\circ} \mathrm{C}$, whereas the boundary condition for all other surrounding surfaces is natural convection with convective coefficient $h=7.5 \mathrm{~W} /(\mathrm{m} \cdot \mathrm{K})$. For internal heat transfer mechanism, radiation is neglected since the maximum temperature is only about $200^{\circ} \mathrm{C}$, so that simulation is based on heat conduction. Local heat generation power by Joule heating of the $\mathrm{SH}$ stress current $\Delta P_{\mathrm{ch}}=I_{d \text {-stress }}^{2} \Delta R_{\mathrm{ch}}$ is applied along the inversion channel (set as $5 \mathrm{~nm}$ in thickness), which increases along the channel since the local channel resistance $\Delta R_{\mathrm{ch}}$ increases from the source to the drain. Temperature dependence of thermal conductivity of related materials [25]-[27] is included, as shown in Table I. Transient thermal analysis shows that upon stress application or removal, the channel temperature rising or falling time to reach a steady state is only about several microseconds, which is far less than the stress duration. The simulated steady-state channel peak temperature depending on stress power is shown in Fig. 14, and in the inset, a typical channel temperature distribution at the gate-oxide/channel interface is shown. An obvious linear dependence of the temperature rise on stress power is obtained. However, data fluctuation also occurs due to variation in power distribution along the channel since devices are stressed under different $V_{d}$ and $V_{g}$ biases. The channel temperature distribution is similar for all stress power conditions. Peak temperature appears close to $(\sim 1.5 \mu \mathrm{m}$ away from) the drain edge, as shown in the figure inset. It is noted that the simulated peak temperature is comparable to a recent work by Takechi et al. [18].

As has been shown in Fig. 2, in the SH degradation, there is an initial stage where device characteristics remain little changed. Significant degradation occurs only after this stage. In Fig. 15, the transfer characteristic of the device subject to initial stress is compared to the unstressed one. A negative parallel shift in the transfer curve, although quite limited, is

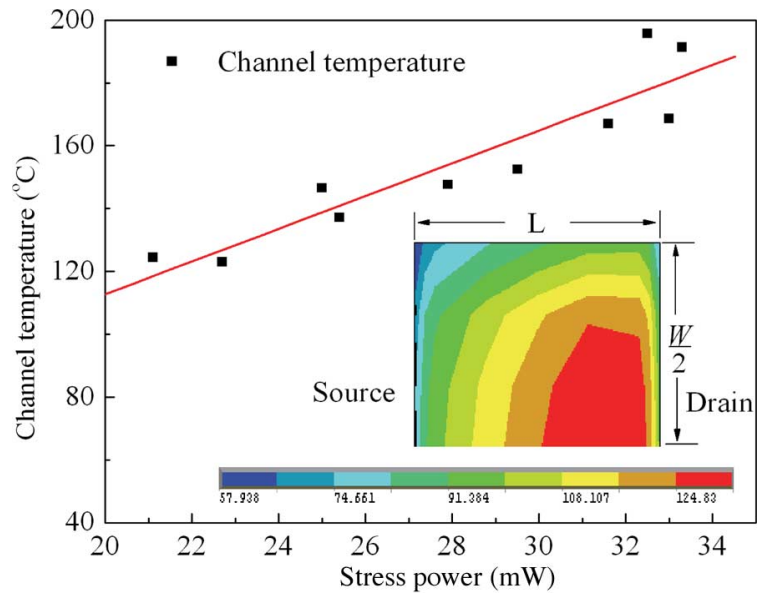

Fig. 14. Simulated channel peak temperature at the gate-oxide/channel interface depending on stress power. The inset is a top view of one half of the channel temperature distribution when the stress power is $27.9 \mathrm{~mW}$.

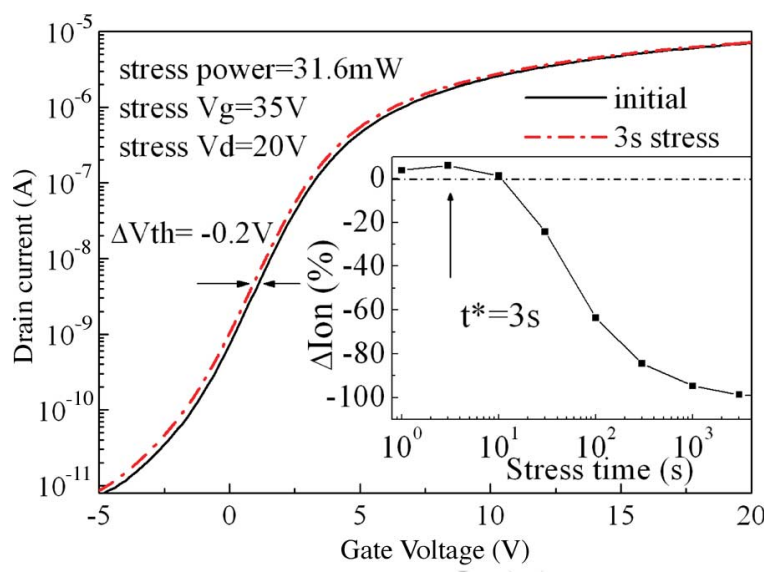

Fig. 15. Comparison of the unstressed device transfer curve to that after the initial stress. A small negative $V_{\mathrm{th}}$ shift is indicated. Inset is $I_{\mathrm{on}}$ variation depending on the stress time for the same device.

clearly observed, indicating a small reduction in the flatband voltage at the initial stage of stress. Correspondingly, a slight initial $I_{\mathrm{on}}$ increase, which is soon overwhelmed by the normal degradation, is also observed. In the figure inset, depending on the transition or turnaround of $I_{\mathrm{on}}$ from the initial stage to the normal degradation stage, a characteristic transition time $t^{*}$ is defined. 


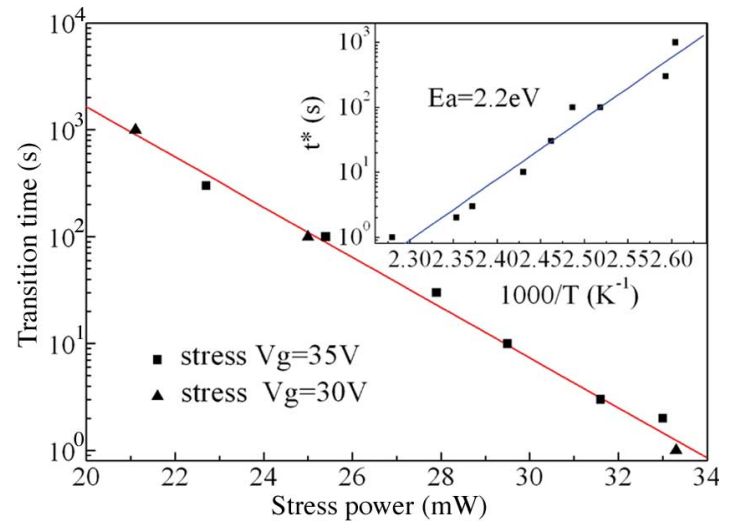

Fig. 16. Dependence of transition time $t^{*}$ on SH stress power. Inset is an Arrhenius plot of the transition time based on the simulated channel temperature rise.

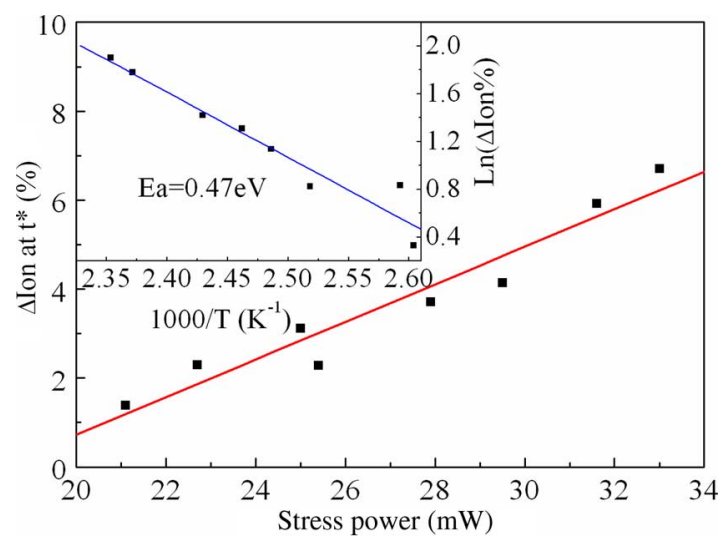

Fig. 17. Dependence of $\Delta I_{\mathrm{on}}$ at transition time on SH stress power. Inset is an Arrhenius plot of the $I_{\mathrm{on}}$ increase at $t^{*}$ based on the simulated channel temperature rise.

As shown in Fig. 16, $t^{*}$ remarkably varies from $\sim 1$ to $\sim 10^{3} \mathrm{~s}$ with decreasing stress power from 33 to $21 \mathrm{~mW}$. Since the respective channel temperature rise is obtained by simulation for each stress power condition, an Arrhenius plot of $t^{*}$ can be illustrated, as shown by the figure inset. The good linearity of the plot indicates that $t^{*}$ is controlled by a mechanism that can be characterized by an activation energy $E_{a}$, which is determined as $\sim 2.2 \mathrm{eV}$ from the slope of the plot. Here, it is worth noting that the channel peak temperature is used to estimate $E_{a}$. The implication is that the channel high-temperature region should control the device degradation characteristics. This assumption is reasonable since most $\mathrm{SH}$-induced defect generation either by dissolution of $\mathrm{Si}-\mathrm{H}$ bonds or by breaking/distortion of $\mathrm{Si}-\mathrm{Si}$ bonds should favorably occur at the channel peak temperature region. In Fig. 17, this turnaround effect is further characterized by the maximum $I_{\text {on }}$ increase during the initial stress, i.e., $\Delta I_{\text {on }}$ at $t^{*}$, which also correlates with stress power. Similarly, its corresponding Arrhenius plot is obtained with good linearity with $E_{a}$ estimated as $\sim 0.47 \mathrm{eV}$.

Although rarely reported in SH degradation, two-stage degradation has been observed by a few groups in other stress conditions, and various mechanisms have been proposed. In dynamic HC stress, Chang et al. [10] reported a $V_{\text {th }}$ twostage degradation, but without $V_{\mathrm{th}}$ negative shift, and attributed the initial degradation stage to breaking of weak $\mathrm{Si}-\mathrm{H} / \mathrm{Si}-\mathrm{Si}$ bonds and the second stage to breaking of strong $\mathrm{Si}-\mathrm{Si}$ bonds. Inoue et al. [16] showed that a combination of ion contamination effect and $\mathrm{SH}$ stress could lead to a similar two-stage degradation. Kouvatsos and Davazoglou [28] observed $V_{\text {th }}$ turnaround on solid-phase crystallization poly-Si TFTs stressed by high $V_{g}$ bias with/without a low $V_{d}$ bias and explained the initial $V_{\text {th }}$ backshift by hole trapping of preexisting oxide and interface traps via a mechanism of electron tunneling out of neutral oxide traps [29]. In HC degradation, Farmakis et al. [12] proposed that hot hole injection followed by hot electron injection can account for such $V_{\text {th }}$ turnaround effect.

In HC two-stage degradation, the initial $V_{\text {th }}$ backshift is accompanied by a $g_{m \_ \text {max }}$ increase as a result of the channel shortening effect [10], [12]. In our observation, the lack of initial $g_{m}$ max increase implies a different underlying mechanism. Based on a previous hypothesis [10], we tentatively propose that at the initial stage of stress, dissolution of $\mathrm{Si}-\mathrm{H}$ and $\mathrm{Si}-\mathrm{OH}$ weak bonds at GBs and the gate-oxide/channel interface first occurs by high current stress with elevated channel temperature under SH. Hence, a small amount of released positive hydrogen ions can diffuse toward the bottom oxide under the influence of the transverse electrical field within the channel, slightly modulating the substrate potential. This floating-body effect [7] could be responsible for the initial small negative shift of $V_{\mathrm{th}}$. In the normal stage of stress, degradation is mainly attributed to defect generation by distorting/breaking of strong $\mathrm{Si}-\mathrm{Si}$ bonds at GBs, resulting in a large increase in $V_{\mathrm{th}}$, which overwhelms the initial $V_{\text {th }}$ backshift.

This explanation is supported by our observation that occurrence of the normal degradation stage $\left(t^{*}\right)$ is controlled by a high $E_{a}$ of $2.2 \mathrm{eV}$, whereas the amount of initial degradation $\left(\Delta I_{\text {on }}\right.$ at $\left.t^{*}\right)$ is characterized by a much lower $E_{a}$ of $0.47 \mathrm{eV}$. Since $E_{a}$ extraction is performed by using simulated channel peak temperatures, it is considered that an estimated $E_{a}$ of $2.2 \mathrm{eV}$ is fairly close to the $\mathrm{Si}-\mathrm{Si}$ bond energy of $1.83 \mathrm{eV}$. It lends further support to the hypothesis that the normal degradation occurs by stress-induced defect generation by breaking/distorting of strong $\mathrm{Si}-\mathrm{Si}$ bonds. Therefore, the carrier trapping mechanism [12], [28], [29] previously proposed to explain the turnaround degradation behavior seems to be not a controlling mechanism in the current observation.

\section{CONCLUSION}

SH degradation of n-type MILC polysilicon TFTs is systematically investigated under different stress powers. All degradation characteristics are found to correlate with stress power, indicating the dominant effect of the channel temperature rise in SH degradation. Degradation slopes of device $V_{\mathrm{th}}, I_{\mathrm{on}}$, and $S S$ suggest a degradation mechanism of defect generation either at GBs or at the gate oxide/channel interface.

A two-stage degradation behavior with turnaround effect at the initial stage is observed. Normal degradation is caused by breaking/distorting of strong $\mathrm{Si}-\mathrm{Si}$ bonds mainly at GBs. The turnaround effect at the initial stage is explained by breaking of weak bonds and releasing positive hydrogen ions, which migrate toward the bottom oxide and modulate substrate 
potential. Based on the simulated channel temperature, $E_{a}$ characterizing both degradation stages is estimated. $E_{a}$ of the initial degradation is found to be much lower than that of the normal degradation.

An anomalous continuous $\mu_{\mathrm{FE}}$ increase along with its $V_{g}$ dependence shift is first observed. The $V_{g}$ dependence shift correlates with device $V_{\text {th }}$ degradation. However, $\mu_{\text {eff }}$ continuously decreases with stress. The anomalous $\mu_{\mathrm{FE}}$ increase is not a true mobility increase, but is a result of the stressinduced $V_{g}$ dependence shift in $\mu_{\text {eff }}$ and the variation of its differential on $V_{g}$. Based on a mobility model in poly-Si TFT, where carrier transport is controlled by $V_{g}$ modulated GB potential barrier, stress-power-dependent GB trap generation is directly observed. All observations are consistent within the same degradation model.

\section{REFERENCES}

[1] Y. Nakajima, Y. Kida, M. Murase, Y. Toyoshima, and Y. Maki, "Latest developments for 'system-on-glass' displays using low-temperature polySi TFTs," J. Soc. Inf. Disp., vol. 12, no. 4, pp. 361-365, 2004.

[2] T. Yoshida, Y. Ebiko, M. Takei, N. Sasaki, and T. Tsuchiya, "Grainboundary related hot carrier degradation mechanism in low-temperature polycrystalline silicon thin-film transistors," Jpn. J. Appl. Phys., vol. 42, no. 4B, pp. 1999-2003, Apr. 2003.

[3] F. V. Farmakis, J. Brini, G. Kamarinos, C. T. Angelis, C. A. Dimitriadis, M. Miyasaka, and T. Ouisse, "Grain and grain-boundary control of the transfer characteristics of large-grain polycrystalline silicon thin-film transistors," Solid State Electron., vol. 44, no. 6, pp. 913-916, Jun. 2000

[4] S. Inoue, M. Kimura, and T. Shimoda, "Analysis and classification of degradation phenomena in polycrystalline-silicon thin film transistors fabricated by a low-temperature process using emission light microscopy," Jpn. J. Appl. Phys., vol. 42, no. 3, pp. 1168-1172, Mar. 2003

[5] F. V. Farmakis, J. Brini, G. Kamarinos, and C. A. Dimitriadis, "Anomalous turn-on voltage degradation during hot-carrier stress in polycrystalline silicon thin-film transistors," IEEE Electron Device Lett., vol. 22, no. 2, pp. 74-76, Feb. 2001.

[6] M. Wong and H. S. Kwok, "High-performance polycrystalline silicon thin-film transistor technology using low-temperature metal-induced unilateral crystallization," Microelectron. J., vol. 35, no. 4, pp. 337-341, Apr. 2004.

[7] Y. Mishima, K. Yoshino, M. Takei, and N. Sasaki, "Characteristics of lowtemperature poly-Si TFTs on Al/glass substrates," IEEE Trans. Electron Devices, vol. 48, no. 6, pp. 1087-1091, Jun. 2001.

[8] L. Handong and S. J. Fonash, "Reliability study of MILC poly-Si TFTs on plastic substrates using postflex transfer process," in Proc. Int. Symp. Thin Film Transistor Technol. VII, 2005, pp. 49-56.

[9] M. Xue, M. Wang, Z. Zhu, D. Zhang, and M. Wong, "Degradation behaviors of metal-induced laterally crystallized n-type polycrystalline silicon thin-film transistors under dc bias stresses," IEEE Trans. Electron Devices, vol. 54, no. 2, pp. 225-232, Feb. 2007.

[10] K. M. Chang, Y. H. Chung, G. M. Lin, C. G. Deng, and J. H. Lin, "Enhanced degradation in polycrystalline silicon thin-film transistors under dynamic hot-carrier stress," IEEE Electron Device Lett., vol. 22, no. 10, pp. $475-477$, Oct. 2001

[11] C. Hu, S. C. Tam, F.-C. Hsu, P.-K. Ko, T.-Y. Chan, and K. W. Terrill, "Hot-electron-induced MOSFET degradation-Model, monitor and improvement," IEEE Trans. Electron Devices, vol. ED-32, no. 2, pp. 375385, Feb. 1985.

[12] F. V. Farmakis, C. A. Dimitriadis, J. Brini, G. Kamarinos, V. K. Gueorguiev, and T. E. Ivanov, "Hot-carrier phenomena in high temperature processed undoped-hydrogenated n-channel polysilicon thin film transistors (TFTs)," Solid State Electron., vol. 43, no. 7, pp. 1259-1266, Jul. 1999.

[13] P. Hermans, R. Bellens, G. Groeseneken, and H. E. Maes, "Consistent model for the hot-carrier degradation in n-channel and p-channel MOSFET's," IEEE Trans. Electron Devices, vol. 35, no. 12, pp. 21942209, Dec. 1988

[14] N. A. Hastas, N. Archontas, C. A. Dimitriadis, G. Kamarinos, T. Nikolaidis, N. Georgoulas, and A. Thanailakis, "Substrate current and degradation of n-channel polycrystalline silicon thin-film transistors," Microelectron. Reliab., vol. 45, no. 2, pp. 341-348, Feb. 2005.
[15] S. Inoue, H. Ohshima, and T. Shimoda, "Analysis of threshold voltage shift caused by bias stress in low temperature poly-Si TFTs," in IEDM Tech. Dig., 1997, pp. 527-530.

[16] S. Inoue, S. Takenaka, and T. Shimoda, "Study of degradation phenomenon due to a combination of contamination and self-heating in polySi thin film transistors fabricated by a low-temperature process," Jpn. J. Appl. Phys., vol. 42, no. 7A, pp. 4213-4217, Jul. 2003.

[17] S. Inoue, H. Ohshima, and T. Shimoda, "Analysis of degradation phenomenon caused by self-heating in low-temperature-processed polycrystalline silicon thin film transistors," Jpn. J. Appl. Phys., vol. 41, no. 11A, pp. 6313-6319, Nov. 2002.

[18] K. Takechi, M. Nakata, H. Kanoh, S. Otsuki, and S. Kaneko, "Dependence of self-heating effects on operation conditions and device structures for polycrystalline silicon TFTs," IEEE Trans. Electron Devices, vol. 53, no. 2, pp. 251-257, Feb. 2006.

[19] M. D. Jacunski, M. S. Shur, and M. Hack, "Threshold voltage, field effect mobility, and gate-to-channel capacitance in polysilicon TFT's," IEEE Trans. Electron Devices, vol. 43, no. 9, pp. 1433-1440, Sep. 1996.

[20] N. Gupta and B. P. Tyagi, "On-current modeling of polycrystalline silicon thin-film transistors," Phys. Scr., vol. 72, no. 4, pp. 339-342, 2005.

[21] H.-L. Chen and C.-Y. Wu, "An analytical grain-barrier height model and its characterization for intrinsic poly-Si thin-film transistor," IEEE Trans. Electron Devices, vol. 45, no. 10, pp. 2245-2247, Oct. 1998.

[22] J. Levinson, F. R. Shepherd, P. J. Scanlon, W. D. Westwood, G. Este, and M. Rider, "Conductivity behavior in polycrystalline semiconductor thin film transistors," J. Appl. Phys., vol. 53, no. 2, pp. 1193-1202, Feb. 1982.

[23] M. Wang, M. Wong, X. Shi, and D. Zhang, "Effective channel mobility of poly-silicon thin film transistors," in Proc. 8th Int. Conf. Solid-State Integr. Circuit Technol., Shanghai, China, 2006, pp. 1395-1397.

[24] M. Wang and M. Wong, "An effective channel mobility-based analytical on-current model for polycrystalline silicon thin-film transistors," IEEE Trans. Electron Devices, vol. 54, no. 4, pp. 869-874, Apr. 2007.

[25] D. G. Cahill, W. K. Ford, K. E. Goodson, G. D. Mahan, A. Majumdar, H. J. Maris, R. Merlin, and S. R. Phillpot, "Nanoscale thermal transport," J. Appl. Phys., vol. 93, no. 2, pp. 793-818, Jan. 2003.

[26] C. J. Glassbrenner and G. A. Slack, "Thermal conductivity of silicon and germanium from $3{ }^{\circ} \mathrm{K}$ to the melting point," Phys. Rev., vol. 134, no. 4A, pp. A1058-A1069, May 1964.

[27] D. G. Cahill, "Thermal conductivity measurement from 30 to $750 \mathrm{~K}$ : The $3 \omega$ method," Rev. Sci. Instrum., vol. 61, no. 2, pp. 802-808, Feb. 1990.

[28] D. N. Kouvatsos and D. Davazoglou, "Gate/drain bias-induced degradation effects in TFTs fabricated in unhydrogenated SPC polycrystalline silicon films," Thin Solid Films, vol. 426, no. 1/2, pp. 250-257, Feb. 2003.

[29] N. Stojadinovic, I. Manic, S. Djoric-Veljkovic, V. Davidovic, S. Golubovic, and S. Dimitrijev, "Mechanisms of positive gate bias stress induced instabilities in power VDMOSFETs," Microelectron. Reliab., vol. 41, no. 9/10, pp. 1373-1378, Sep./Oct. 2001.

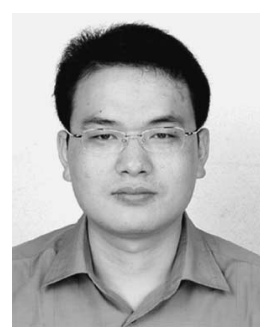

Huaisheng Wang was born in Chaohu, China, in 1979. He received the B.S. degree in electronic engineering from Hefei University, Hefei, China, in 2001. $\mathrm{He}$ is currently working toward the M.S. degree at the Department of Microelectronics, Soochow University, Suzhou, China.

He was with Huaian Information College, Huaian, China, until 2005. His current research work is about device degradation behavior of poly-Si TFT under self-heating or hot carrier stress.

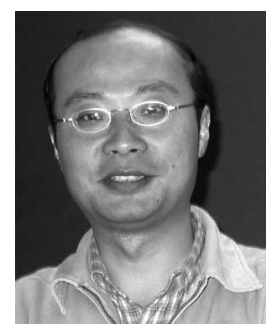

Mingxiang Wang (M'07) received the B.S. degree in physics and the $\mathrm{Ph} . \mathrm{D}$. degree in condensed matter physics from Nanjing University, Nanjing, China, in 1993 and 1998, respectively.

From 1998 to 2001, he was a Postdoctoral Research Associate with the Department of Electrical and Electronic Engineering, The Hong Kong University of Science and Technology, Kowloon, Hong Kong. Then, he joined Semiconductor Manufacturing International Corporation, Shanghai, as a Member of the technical staff in process reliability engineering. He is currently with the Department of Microelectronics, Soochow University, Suzhou, China. His research interests include thin-film materials and devices, semiconductor device physics, and device reliability. 


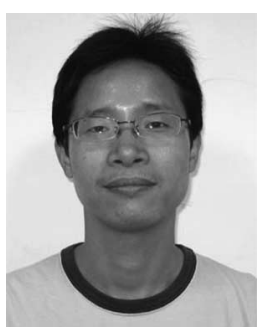

Zhenyu Yang was born in Wuxi, China, in 1979. He received the B.S. degree in physics and the M.S degree in microelectronics from Soochow University, Suzhou, China, in 2002 and 2007, respectively.

$\mathrm{He}$ is currently with the Department of Microelectronics, Soochow University, working on the field of IC reliability.

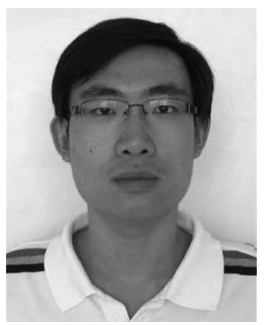

Han Hao was born in Xuzhou, China, in 1982. He received the B.S. degree in microelectronics from Soochow University, Suzhou, China, in 2005. He is currently working toward the M.S. degree at the Department of Microelectronics, Soochow University.

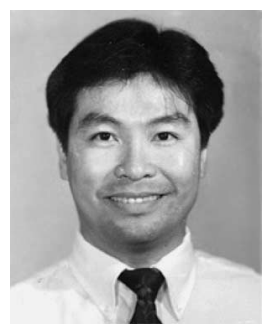

Man Wong (S'83-M'88-SM'00) was born in Beijing, China. He received the B.S. and M.S. degrees from the Massachusetts Institute of Technology, Cambridge, in 1979 and 1984, respectively, and the Ph.D. degree from Stanford University, Stanford, CA, in 1988, all in electrical engineering.

From 1985 to 1988 , he was with the Center for Integrated Systems, Stanford University, where he worked on tungsten-gate MOS technology. From 1988 to 1992 , he was with the Semiconductor Process and Design Center, Texas Instruments, Dallas, and worked on the modeling and development of integrated-circuit metallization systems and dry/vapor surface-conditioning processes. In 2003 he was appointed an Honorary Guest Professor of Nankai University, Tianjin, China. He is currently with the Department of Electrical and Electronic Engineering, The Hong Kong University of Science and Technology, Kowloon, Hong Kong. His research interests include microfabrication technology, device structure, and material; physics and technology of thin-film transistor; organic light emitting diode display technology; and modeling and implementation of integrated microsystems.

Dr. Wong is a member of Tau Beta Pi, Eta Kappa Nu, and Sigma Xi. 